\begin{tabular}{|l|l|l||}
\hline \multicolumn{2}{|c|}{ PublisherInfo } \\
\hline \hline PublisherName & $:$ & BioMed Central \\
\hline \hline PublisherLocation & $:$ & London \\
\hline \hline PublisherImprintName & $:$ & BioMed Central \\
\hline \hline
\end{tabular}

\title{
Fruitfly fucosylation
}

\begin{tabular}{|l|l|l||}
\hline \multicolumn{2}{|c|}{ ArticleInfo } \\
\hline \hline ArticleID & $:$ & 4332 \\
\hline \hline ArticleDOI & $:$ & $10.1186 /$ gb-2002-3-4-reports0021 \\
\hline \hline ArticleCitationID & $:$ & reports0021 \\
\hline \hline ArticleSequenceNumber & $:$ & 22 \\
\hline \hline ArticleCategory & $:$ & Paper report \\
\hline ArticleFirstPage & $:$ & 1 \\
\hline \hline ArticleLastPage & $:$ & 4 \\
\hline \hline & & RegistrationDate : 2002-2-12 \\
ArticleHistory & $:$ & Received \\
\hline \hline ArticleCopyright & $:$ & BioMed Central Ltd2002-2-12 \\
\hline \hline ArticleGrants & $:$ & \\
\hline \hline
\end{tabular}




\begin{tabular}{|l|l|l||}
\hline ArticleContext & $:$ & 130593344 \\
\hline
\end{tabular}

\section{Wim D'Haeze}

\section{Abstract}

A biocomputational study of the fruitfly genome reveals the presence of new genes putatively involved in fucosylation

\section{Significance and context}

Glycans - the oligosaccharide chains attached to many extracellular and membrane proteins and lipids - are of importance in a variety of biological processes. $N$-glycans are, for instance, involved in protein folding, glycosylated proteins have important roles in fertilization and pattern formation during embryogenesis, and in some instances $O$-glycans are involved in cellular signaling, often leading to the regulation of transcription and translation. Glycans often contain the sugar fucose as an essential component. The A, B, and $\mathrm{O}$ blood-group antigens, for instance, contain $\beta 1,2$-fucosylated lactosamine, and fucosylated proteins are important for the normal development of an organism. Fucosylation requires GDP-L-fucose as a donor and also the presence of particular fucosyl transferases, which transfer the fucosyl residue from the donor to the acceptor molecule. GDP-L-fucose can be synthesized through a de novo pathway from GDP-mannose or through the salvage pathway from fucose. The removal of fucosylated glycans is mediated by fucosidases, and defects in both fucosylation and fucose removal lead to inherited disorders in humans, for example, fucosidosis, a recessive autosomal disorder characterized by an impaired lysosomal degradation of fucosylated glycans. Roos et al. carried out a biocomputational study on the genome of the model organism Drosophila melanogaster to characterize the metabolic pathways involving fucosylated glycans, which may contribute to a better understanding of similar processes in humans.

\section{Key results}

From their in silicostudy, Roos et al. identified two novel enzymes in D. melanogaster, similar to a GDP-mannose-4,6-dehydratase and a GDP-4-keto-6-deoxy-D-mannose epimerase/reductase, which are proposed to be involved in the synthesis of GDP-L-fucose. From this, they showed that, in $D$. melanogaster, GDP-L-fucose is formed solely through the de novo synthesis pathway, involving a GDPmannose-4,6-dehydratase and a GDP-4-keto-6-deoxy-D-mannose epimerase/reductase. D. melanogaster most probably lacks the salvage pathway for synthesizing GDP-L-fucose, as no genes similar to the mammalian genes encoding fucokinase and fucose-1-phosphate guanylyltransferase could be found. 
Study of the fruitfly genome further revealed the presence of genes for two novel fucosyltransferases ( $\beta 1,3$ - and an $\beta 1,6$-fucosyltransferase), two $O$-fucosyltransferases, proposed to be involved in the direct fucosylation of proteins at serine/threonine residues, and a fucosidase. Although $\beta 1,2$-fucosyltransferases are present in some bacteria and mammals, no genes encoding these enzymes were found in D. melanogaster. On the basis of the study of the D. melanogaster genome sequence and homology searching, two new human fucosyltransferases - closely related to $\beta 1,3$ / $\beta 1,4$-fucosyltransferases - were identified.

\section{Links}

Information about the genome sequence of D. melanogaster is available at the Berkeley Drosophila Genome Project web page .

\section{Conclusions}

Roos et al. conclude that new members of protein families can be identified in one organism by their similarity to genes identified in other organisms.

\section{Reporter's comments}

Although expressed sequence tags are available for many of the glycan metabolism genes that Roos et al. discovered in D. melanogaster, suggesting that they are transcribed, their role in the production of fucosylated compounds still remains to be proved. The construction of precise knockouts and/or detailed biochemical studies and assays will be needed to confirm their proposed role. Such studies will be of importance in understanding these pathways in humans and thus in developing therapies for patients with defects in fucosylation metabolism.

\section{Table of links}

Journal of Biological Chemistry

Berkeley Drosophila Genome Project web page 


\section{References}

1. Roos C, Kolmer M, Mattila P, Renkonen R: Composition of Drosophila melanogaster proteome involved in fucosylated glycan metabolism. J Biol Chem. 2002, 277: 3168-3175. 0021-9293

This PDF file was created after publication. 